\title{
423
}

\section{EL ARCHIVO DIGITAL}

COMO OBJETO

DE ESTUDIO PARA

COMPRENDER

MECANISMOS DE

SUBJETIVACIÓN QUE

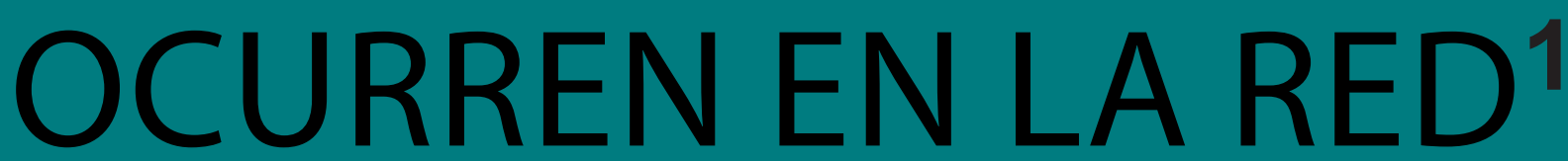

Pablo Ignacio Vallejos Baccelliere

Magíster en Literatura - Investigador Independiente 


\section{$4526=$}

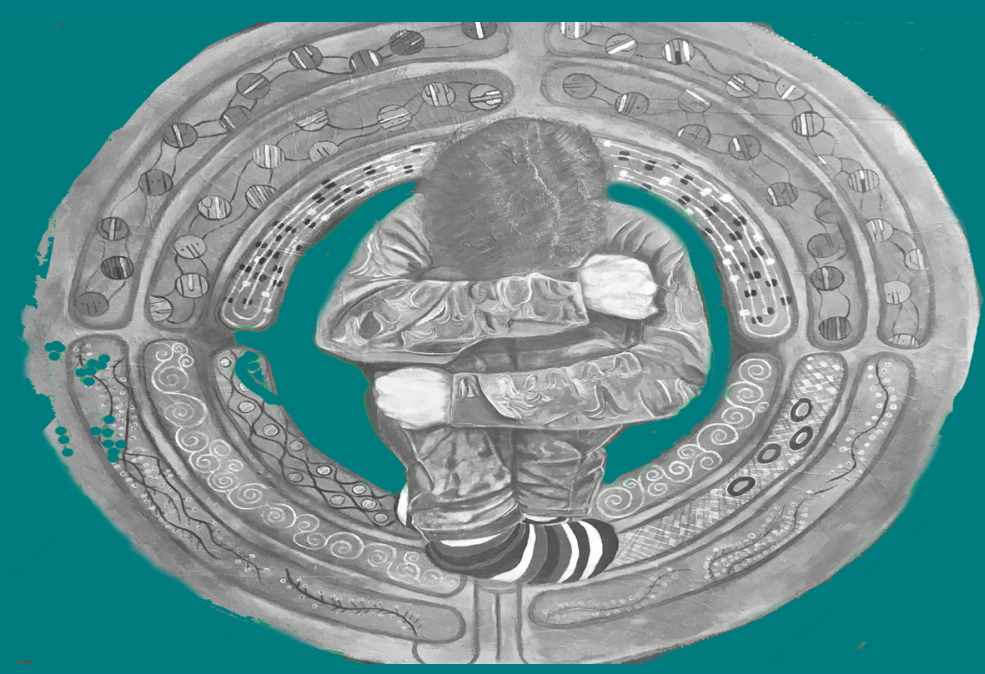

Resumen || Para analizar lo digital como objeto de estudio es necesario establecer una base teórica en las humanidades, de modo que se pueda comprender qué cambios y reconfiguraciones sufren las culturas, las sociedades y el arte. En ese sentido, el presente artículo toma el concepto de la subjetividad y lo sitúa en el contexto de la conexión a internet masiva y el uso de aparatos electrónicos, para comprender sus condiciones de existencia. Lo que aquí se desarrolla se puede extrapolar a diferentes entramados conceptuales complejos que se reconfiguran en las lógicas de internet y las nuevas tecnologías de la información y la comunicación, permitiendo así pensar lo digital a un nivel general, comprendiendo una base de conceptos necesarios para analizar las humanidades y el arte en la cultura digital. Se establece, entonces, un entramado teórico conceptual apoyado por algunos ejemplos pertinentes de obras y situaciones específicas, para ofrecer nuevos puntos de vista reflexivos en torno a teorías culturales y literarias. Asimismo, se explorarán las problemáticas que suscitan las nuevas tecnologías y su relación con los usuarios y los objetos artísticos.

\section{Palabras clave || Humanidades | Subjetividad | Cultura digital | Usuario | Técnica}

Abstract || In order to analyze the digital as an object of study it is necessary to establish a theoretical basis in the humanities, to understand the changes and reconfigurations that cultures, societies and the arts have experienced. As such, the aim of this article is to situate the concept of subjectivity in the context of the massive Internet connection and the use of electronic devices, to comprehend how it is possible in the digital. Thus, this thesis can be extrapolated to different conceptual frameworks, which are reconfigured under electronic and networked environments, and new information and communication technologies. This allows a critical study of the digital on a general level, comprehending a base of concepts that are needed to analyze the humanities and the arts in the digital cultures. A theoretical-conceptual framework is established, alongside examples of artistic works and real-life situations, so new points of view are added to literary and cultural theories. Likewise, this article will explore the issues present in the relations between users, artistic objects and new technologies.

\section{Keywords || Humanities | Subjectivity | Digital culture | User | Technic}

Resum I| Per a analitzar el digital com a objecte d'estudi és necessari establir una base teòrica en les humanitats, de manera que es pugui comprendre quins canvis i reconfiguracions sofreixen les cultures, les societats i l'art. En aquest sentit, el present article pren el concepte de la subjectivitat i el situa en el context de la connexió a Internet massiva i l'ús d'aparells electrònics, per a comprendre les seves condicions d'existència. El que aquí es desenvolupa es pot extrapolar a diferents entramats conceptuals complexos que es reconfiguren en les lògiques d'Internet i les noves tecnologies de la informació i la comunicació, permetent així pensar el digital a un nivell general, comprenent una base de conceptes necessaris per a analitzar les humanitats i l'art en la cultura digital. S'estableix, doncs, un entramat teòric conceptual secundat per alguns exemples pertinents d'obres i situacions específiques, per a oferir nous punts de vista reflexius entorn de teories culturals i literàries. Així mateix s'exploraran les problemàtiques que susciten les noves tecnologies i la seva relació amb els usuaris i els objectes artístics.

Paraules clau || Humanitats | Subjectivitat | Cultura digital | Usuari | Tècnica 


\section{Introducción: el sujeto en la era digital}

La tecnología en el último tiempo, más o menos desde el final de la segunda guerra mundial hasta el día de hoy, se ha modificado y perfeccionado a ritmos cada vez más veloces. Un teléfono inteligente o una computadora portátil de alta potencia eran fantasías hace treinta años. Hoy, la tecnología afecta a las sociedades y a las culturas de diferentes formas; principalmente en relación con los centros de poder, el mercado global y las estructuras sociales, por una parte, y con la construcción de subjetividades, individuos y comunidades culturales, por otra. Así, no se puede negar la injerencia que tienen las tecnologías de la información y la comunicación (TIC de aquí en adelante) en las sociedades y la cultura. Es más, actualmente se habla de la existencia de la cultura digital, la cual a la vez condiciona a las personas y los grupos humanos, como también es condicionada por los mismos, en un proceso de retroalimentación de información y flujos de datos, puesto que los algoritmos se adaptan a los usuarios para reconfigurar la red.

Es este el contexto en el que conceptos como la subjetividad se tornan aún más complejos de lo que ya son. A las corrientes antihumanistas ${ }^{2}$ se les suman las teorías digitales de la cultura, el poshumanismo, el transhumanismo, entre tantas otras. Por ello, la pregunta que motiva al presente artículo se plantea el lugar del sujeto en la relación entre el ser humano y la tecnología. Llamar usuario a una persona que utiliza una TIC implica que este sujeto se relaciona con el mundo a través de tecnologías y mecanismos electrónicos y digitales, lo cual conlleva cambios en cuanto a la relación de la persona con el mundo, con otros y consigo misma. Por su parte, el lector o el espectador y su relación con una obra se transforman bajo la lógica del usuario, en donde el lector activo potencia su capacidad de intervención de según los límites establecidos por los artistas.

De este modo, en el presente artículo no se pretende agregar una definición más al concepto de subjetividad, sino que se desarrollarán sus condiciones de existencia en entornos digitales, así como también su relación con el usuario de una tecnología. Esta idea nace de la necesidad de pensar críticamente sobre lo digital como objeto de estudio. Esto quiere decir que no se trata de utilizar conceptos clásicos sobre temáticas, objetos y sujetos digitales, sino de actualizarlos para así entregar nuevas formas de aproximarse a lo digital, desde lo digital. Es necesario no sólo llevar las humanidades a nuevos territorios, sino ser consciente del lugar de uno mismo como usuario de diferentes tecnologías (desde teléfonos inteligentes hasta tarjetas bancarias, por ejemplo). En ese sentido, las ideas desarrolladas en este artículo siguen la línea de la «ecología de conocimiento» planteada por Élika Ortega (2019). A grandes rasgos, la autora describe las problemáticas de estudiar

\section{NOTAS}

1| El presente artículo es parte de la investigación realizada para mi tesis de magíster «El sujeto-archivo digital: procesos arcónticos de subjetivación en la red», financiada por el proyecto Fondecyt Regular N 1180771 , «Cartografías de la literatura digital latinoamericana», dirigido por Carolina Gainza C.

2 | Corrientes de pensamiento que cuestionan el concepto de humanismo de la ilustración, el cual ha influenciado la mayoría de la filosofía de los últimos siglos. Según estas corrientes, el ser humano definido por el humanismo tradicional hace referencia a un «hombre blanco heterosexual», incluso más específicamente europeo. Por ello, los pensadores del antihumanismo son aquellos que han abogado por el feminismo, el poscolonialismo, el poshumanismo, etc. 
lo digital desde un academicismo cargado de centralismo en cuanto a culturas y discursos hegemónicos occidentales (europeos y estadounidenses). Por ello explica la necesidad de generar humanidades digitales desde lo interdisciplinar, la multiculturalidad y la generación de conocimiento autocrítico. El intelectual, en este sentido, no puede desligarse de su condición de sujeto inserto en un marco global digital que dialoga con sus propias estructuras culturales, sociales y hegemónicas locales.

Hablar de «lo digital» es amplio y es probable que aún no se logren definiciones claras sobre lo que implica. Sin embargo, preguntarse por cómo, cuándo y por qué tiene lugar da como fruto reflexiones teóricas que apuntan precisamente a indicar qué es lo que se debe estudiar sobre lo digital. Aproximarse así a este objeto de estudio permite nuevas miradas en muchas áreas de las humanidades, entre ellas los estudios literarios, puesto que conceptos como el autor, el lector y los procesos de escritura son tensionados en nuevas materialidades y formas de configurarse, como por ejemplo los hipermedios en internet, la representación del código y su programación.

A su vez es necesario revisar el concepto de "archivo», debido a su implicancia en las tecnologías informáticas y la importancia que tienen las prácticas archivísticas en las humanidades y la literatura. En informática, el archivo es un conjunto de datos específicos que producen una unidad, la cual es representada en la pantalla de una tecnología. Es el código con el cual un usuario interactúa. Sin embargo, hoy más que nunca, hay programaciones y algoritmos configurados para producir microarchivos con la información de las personas que utilizan un aparato electrónico, por lo cual para entender el concepto de subjetividad en lo digital es necesario relacionarlo con mecanismos archivísticos que resultan en procesos de subjetivación contemporáneos. Además, surgen problemáticas sobre la privacidad, el acceso a archivos digitales y las formas en que inciden en las configuraciones sociales. El concepto de archivo digital necesita ser entendido desde su contraparte analógica, para así entender los mecanismos generales que inciden en su producción y en los procesos de subjetivación que conllevan. En el segundo apartado del presente artículo se discutirá la relevancia de lo planteado por Jacques Derrida en Mal de archivo (1997), contraponiéndolo a teorías contemporáneas sobre el concepto en su sentido tanto digital como analógico. La decisión de utilizar el concepto en ambos sentidos proviene de cómo la recopilación de datos produce discursos y subjetividades según las tecnologías de una época específica. Además, el archivo digital contemporáneo se construye sobre las bases de lo que autores como Derrida o Foucault ya habían mencionado antes de la rápida evolución de las TIC tal como se conocen hoy en día. 
El objetivo de este artículo es proponer una conceptualización sobre el archivo como mecanismo de subjetivación en entornos digitales, tomando en cuenta las lógicas del archivo analógico y su evolución hacia el archivo digital. Esto será complementado mediante el análisis de obras y proyectos literarios, como también iniciativas que se están dando desde las humanidades digitales. Así surgen preguntas relacionadas con ramas específicas como la literatura: ¿qué sucede con el usuario/lector al ser subjetivizado mediante procesos digitales? ¿Cómo se transforma la producción literaria y las subjetividades que la rodean en la red? Estas preguntas suscitan reflexiones que serán desarrolladas a lo largo del presente artículo.

Finalmente, es necesario señalar que este artículo entiende que la subjetividad se sitúa históricamente para ser producida, y que el cuerpo sobre el cual los mecanismos de subjetivación son implementados es de vital importancia para entender la relación entre el ser humano y las tecnologías. No se debe pensar que lo digital existe en contraposición a lo físico, o como Rey y Boesel (2014) plantean: lo online y lo offline son parte de una misma realidad y en conjunto configuran las subjetividades dentro y fuera de lo digital. Por eso, el archivo analógico no es reemplazado por el digital, sino que es complementado por este. De este modo, «the contemporary subject is therefore an embodied subject, one whose materiality resides not in any one distinct and separate médium [...], but which is performed both within and across multiple media» (2014: 178), siendo estos medios tan diversos como el propio cuerpo, la tecnología y lo digital. Por ello, parafraseando ahora a Rob Gallagher (2018), el sujeto digital se construye por medio de diversos mecanismos y formas de producción del discurso. Por una parte, en lo digital se le clasifica según diversos mecanismos de recopilación de datos informáticos. Por otra, es mediante estos mecanismos como cada sujeto configura una presencia de sí mismo en entornos digitales, conformando la «subjetividad aumentada» que plantean Rey y Boesel. Ahora bien, según Gallagher esto implica la supresión de la biografía narrativa por la implementación de archivos digitales. Sin embargo, y a modo de cierre de este apartado, es importante destacar que tanto el archivo como la construcción narrativa de subjetividades son dos mecanismos que se entrelazan para la producción de subjetividades en la era digital.

\section{Algunos planteamientos sobre la subjetividad}

Es menester situar un concepto tan amplio como el de subjetividad desde un entramado teórico, para así desglosar sus mecanismos de producción en la red. En primer lugar, en este artículo se entiende que el sujeto es un producto, el cual resulta de las técnicas que el 
poder utiliza para controlar, disciplinar y configurar a las personas de una sociedad determinada. Estas técnicas son las llamadas de subjetivación, y son comunes en diferentes momentos históricos. La línea que se seguirá en este artículo es la planteada a lo largo de extensas obras de autores como Foucault. A grandes rasgos, las técnicas de subjetivación son mecanismos aplicados desde el poder hacia los cuerpos de los seres humanos para definirlos como sujetos insertos en una sociedad específica. Por ejemplo, para Foucault, en Vigilar y castigar, la examinación de los individuos los ingresa por medio de la escritura al discurso, clasificándolos según categorías que los construyen como sujetos (Foucault, 2002: 194). Por su parte, Deleuze y Guattari, en Mil mesetas, explican lo que ellos entienden por el «capital», definiéndolo como un «punto de subjetivación que constituye a todos los hombres en sujeto, pero unos, los capitalistas, son sujetos de enunciación, mientras que otros, los proletarios, son sujetos de enunciado sujetos a máquinas técnicas» (Deleuze y Guattari, 2002: 462). De este modo, es en el discurso donde se construyen los mecanismos de subjetivación. Las tecnologías que llevan a cabo estos mecanismos se relacionan con la escritura y la inscripción de los cuerpos en los entramados de poder. Así, como se analizará más adelante con el archivo, se produce el discurso a la par de la producción de sujetos según los órdenes sociales que preexisten dicha producción.

¿Cómo extrapolar lo planteado por los filósofos mencionados al contexto digital? Mercedez Bunz es una pensadora contemporánea que en su libro La utopía de la copia sitúa las técnicas de subjetivación en entornos digitales conectados a internet. La autora reflexiona sobre el lugar del sujeto en un capítulo que precisamente se titula «El sujeto y la red». En él se subraya la importancia de la huella y lo relaciona con las cookies de un navegador de internet (Bunz, 2007). Las cookies son un pequeño conjunto de datos enviados por un sitio web, almacenados en el navegador del usuario, de manera que el sitio web puede consultar la actividad previa del navegador. En resumidas cuentas, es aquello que indica la presencia digital del usuario, la cual no necesita el cuerpo físico del sujeto predigital para expresarse. Es la información recopilada algorítmicamente sobre los hábitos de un usuario al utilizar un navegador de internet. En dicho capítulo se explica que «la huella del sujeto es hecha visible cuando se programa al sujeto dentro de la tecnología en la red, es decir, a posteriori [sic]» (2007: 50). La huella es interpretada mediante la interacción tecnológica, estableciendo una organización o unidad entre ella y la experiencia usuario. Cuando la huella es hecha visible, el usuario deviene en sujeto dentro de la red. Es decir, bajo las lógicas de lo digital, uno es un sujeto en la medida que se presenta dentro del medio. Sin embargo, el proceso de subjetivación en sí mismo sucede previo a la presentación del sujeto digital y ocurre en un instante al que se llamará algorítmico. En este instante, la 
persona de carne y hueso se expresa mediante la huella, condición de posibilidad de aparición del sujeto en la red. En el actual contexto de la cultura digital se establece una

\footnotetext{
tecnología que constituye al sujeto relacionándose con él como su otro. Este concepto de la diferencia pone en primer plano el hecho de que no podría haber ninguna unidad, ninguna identidad sin otro [...] el sujeto no es sometido a la técnica pero tampoco le es indiferente. Está unido a la técnica por un sistema de funciones que pueden ser descritas siempre y cuando sus diferentes niveles sean cuidadosamente diferenciados (2007: 78).
}

La técnica tiene la capacidad de producir al sujeto por medio de la inscripción de sus huellas. Es un proceso de consignación informático mediado por algoritmos programados para captar cierto tipo de información específica. Según la autora, las TIC e internet configuran un otro que marca la diferencia entre el usuario y la red, entre la persona y sus propias huellas. La subjetividad del usuario, en este sentido, es coproducida tanto por la máquina como por el ser humano, debido a que la archivación algorítmica, en su carácter «en tiempo real», específico y momentáneo, se actualiza constantemente en el proceso de producción de la diferencia. El discurso introduce la figura del sujeto en el medio, idea bajo la cual Bunz analiza este proceso en el contexto de los discursos programados y algorítmicos, de los códigos numéricos y los valores cuantitativos.

En resumen, el sujeto no se condice con el cuerpo del usuario, sino que es un otro programado dentro de la tecnología, en el interior de internet. Por ello existe la posibilidad de producir subjetividades "a la medida» de cada usuario, las cuales se multiplican en cada nuevo comando activado en la red. La tecnología descrita hasta ahora es la de los algoritmos diseñados para captar las huellas de los usuarios, codificar y digitalizar la presencia de este en números cuantificables, generando así una subjetividad previa a la presencia, una protosubjetividad definida como un conjunto de datos que las máquinas y las estructuras digitales interpretan como el usuario. Así, pues, se cierra este apartado y se introduce el siguiente, puesto que es necesario desarrollar brevemente el concepto de archivo digital y cómo las subjetividades digitales se configuran a partir de este.

\section{El archivo digital como mecanismo de subjetivación}

En Mal de archivo, Jacques Derrida desarrolla el concepto de archivo entendido como un mecanismo de producción de hegemonías y personas, inserto en una sociedad específica, cuyos procedimientos de documentación y resguardo configuran y producen aquello mismo que se archiva. El archivo, para el filósofo francés, cumple las funciones de «origen» y «mandato». Esto es: la fijación de 
un punto en el pasado común de un grupo humano, un punto de «origen» histórico o mito fundacional, mediante el cual se construye una sociedad homogénea en su sentido amplio. Para mencionar ejemplos basta con observar en qué medida los sentimientos de patriotismo se relacionan con un «deber-ser» que nace de una interpretación histórica de la tradición, las costumbres y la cultura. Andrés Maximiliano Tello, en su libro Anarchivismo, define el concepto de archivo de la siguiente manera: es un «producto heterogéneo de un conjunto de relaciones y tensiones sociales mucho mayor, cuya condición de posibilidad está dada por una concatenación de cuerpos y fuerzas que no responden en ningún caso a una organización social determinada por naturaleza» (Tello, 2018: 27). En ese sentido, tomando mucha inspiración de Derrida, Tello define el concepto contrario a lo que podría ser una creencia popular: no se trata de un organismo que recopila documentos referentes a la historia y las estructuras culturales de una sociedad, sino que eso sucede de modo superficial. La verdadera misión del archivo es la de seleccionar qué documentos pertenecen a una sociedad o cultura específica, qué discursos se deben conservar y, por consecuencia, qué debe ser omitido o eliminado. El archivo es violento, puesto que selecciona qué se deja fuera del conjunto de documentos que lo conforman. La clasificación y la selección son acciones de un poder específico: el poder arcóntico ${ }^{3}$ encarnado por diversos organismos e instituciones a lo largo de la historia. Hoy, siguiendo a Tello, se puede hablar de un capitalismo arcóntico, es decir, la archivación de todo ámbito humano y de producción social, por ende, la capitalización de la información.

La violencia de archivo y sus mecanismos de configuración no se extraen de sus documentos, sino que, tal como menciona Ann Stoler en The Archival Grain, interesa más el archivo como un proceso que como un objeto, en la medida que en su estructura da cuenta de múltiples elementos que conformaban la sociedad de una época específica (Stoler, 2010). De este modo, analizando las tecnologías de archivo y sus procesos de producción, se pueden extraer tensiones sociales, mecanismos burocráticos, ansiedades políticas, prácticas, costumbres e incluso discursos hegemónicos. A su vez, este artículo sigue las reflexiones de Ward y Wismicki en "The Archive after Theory», en donde se plantea la problemática de definir el archivo poscolonial bajo la sombra del archivo «imperial». Estos autores desarrollan la necesidad de nuevas formas de construir archivos que sean críticos en sí mismos (Ward y Wismicki, 2019). De cierto modo, se trata de archivos transparentes que expongan aquello que Stoler resalta de sus investigaciones: el contexto de producción. Los autores indican la posibilidad de los «archivos especulativos» los cuales son cocreados en un sentido de comunidad, siguiendo mecanismos no imperialistas o hegemónicos. Este planteamiento dialoga completamente con el concepto de «anarchivo», el cual

\section{NOTAS}

3| El arconte, según Derrida, es la figura encargada de resguardar el archivo, además de seleccionar los documentos que lo conforman. En consonancia con las ideas del filósofo, la institución produce tanto el discurso como los sujetos que ingresan en él. De este modo, el poder arcóntico es la fuerza aplicada desde la disciplina y/o el control sobre los cuerpos según las lógicas del archivo (recopilación de información y acceso, por mencionar algunas). Por ello también se puede hablar del «capitalismo arcóntico», el cual es el mecanismo de circulación, acumulación y valorización del capital en la actualidad. 
según Antonio Lafuente puede definirse de la siguiente forma: «el archivo sueña con estabilizar el mundo, el anarchivo nos promete desorganizarlo. En las entrañas del archivo viven los historiadores, los jueces y los auditores, en las del anarchivo proliferan los poetas, los rebeldes, los cómicos» (Lafuente, 2015). ¿Puede el archivo digital dar pie a un archivo cocreado, un anarchivo? ¿Cómo se ha actualizado el poder arcóntico en la era digital?

Hasta comienzos del siglo XXI, las subjetividades estaban condicionadas por los valores atribuidos a ciertas categorías como, por ejemplo, las mujeres, los ancianos, los obreros y los niños, entre tantas otras. Sin embargo, en lo digital, el archivo toma nuevas formas y técnicas para afectar los cuerpos y controlar a las poblaciones. Manuel de Landa, en su artículo "The Archive Before and After Foucault» explica que "what was innovate about the new archives was precisely that they objectified individuals not as members of a pre-existing category, but in all their uniqueness and singularities» (De Landa, 2003: 11). En ese sentido, se puede afirmar que la clasificación también se estructura en procesos de retroalimentación, ya no ligada del todo a parámetros establecidos por el poder arcóntico, sino afectada por los hábitos de los usuarios. Es decir, en el momento de utilizar una TIC, un usuario ingresa los datos y las huellas que dan inicio a los procesos de producción del archivo. Este archivo es único en cuanto cada experiencia en la red también lo es. De este modo, por medio de algoritmos digitales, las experiencias futuras de los usuarios se ven condicionadas por lo que se interpreta de estos archivos (hábitos digitales, preferencias, etc.). Por ello, el cambio desde los sistemas hegemónicos del pasado reciente hasta la actualidad de las tecnologías digitales, radica en cómo los mecanismos de subjetivación archivísticos se potencian en la red por medio de las huellas del usuario y su experiencia singular. De todas formas, esta singularidad es aparente puesto que, si bien varios algoritmos son programados para captar información y generar enlaces de acuerdo a las interacciones anteriores del usuario específico, los intereses de una persona que utiliza internet están condicionados por diferentes sistemas: el mercado, el Estado, la vigilancia, entre otros.

Dice Tello: «la acumulación capitalista se reconfigura hoy mediante la desapropiación que opera en el registro de los "bienes inmateriales", es decir, mediante la privatización de huellas de la existencia común» (2018: 246). Se puede afirmar que existe un nivel de microarchivos personales que subjetivizan al usuario para sí mismo y su relación con otros, y mecanismos macroarchivísticos que captan a los sujetos y los construyen de acuerdo a unos u otros intereses económicos y políticos. Finalmente, los intereses de cada uno están elaborados de antemano por la oferta de opciones del mercado del capitalismo tardío. Por ejemplo: los macroarchivos 
son conformados por la información que configura una sociedad o una cultura. No se trata sólo de museos, bibliotecas o leyes, sino también, sobre todo hoy en día, de cómo la información personal de cada usuario es utilizada por grandes empresas de comunicación e información en pos de la vigilancia, por una parte, y la potenciación del mercado generando las opciones de consumo. Por su parte, se habla de microarchivos para referirse a los datos personales de cada usuario en su singularidad, los cuales no sólo son la información que conscientemente se comparte en internet, sino también, como se dijo anteriormente, las huellas y los hábitos de uso, producción y consumo de la información.

Para el filósofo de medios Wolfgang Ernst «the internet constitutes a new type of transarchive», es decir archivos hiperconectados en permanente actualización, donde

net archives are a function of their software and transmission protocol rather than of content, to which technology is indifferent. Beyond the archival principle of provenance, the internet's cybernetic dispositive itself operates as a command system (arché) far more time critical than classical archives ever were (Ernst, 2013: 84).

La organización del contenido archivable ya no recae en su clasificación, sino que es comandado mediante las lógicas de la transmisión de datos masivos, organizando así los flujos de información que conforman la red. El mandato de Derrida hoy en día, siguiendo a Ernst, puede actualizarse con la palabra «comando», la cual significa tanto una orden específica y radical, como también la instrucción que un usuario ejecuta sobre un sistema informático. El comando se ejerce sobre el usuario y la máquina en el proceso de la archivación digital, en cuanto la máquina transmite los datos según la orden, y el usuario genera lo que por ahora podría llamarse un bloque de datos o su información en un momento específico. En su sentido amplio, internet procesa la transmisión de datos sin efectuar una selección a priori.

El archivo es generado una y otra vez durante la interacción entre una persona y una TIC.

As a machinic net of finite automata, the internet has no organized memory and no central agency, defined rather by the circulation of discrete states. If there is memory, it operates as a radical constructivism: always just situationally built, with no enduring storage (2013: 138).

Sin embargo, Ernst está pensando en cómo se construye la memoria del usuario en su interacción con la máquina, por lo que habría que preguntarle al autor qué sucede con los servidores que posibilitan el acceso y la transmisión de datos, a la vez que pueden registrarlos y acumularlos, los cuales tienen un espacio físico delimitado 
ligado a un contexto geopolítico. El data mining actúa de forma retroalimentaria, debido a que las corporaciones y organismos de vigilancia que efectúan los procesos de consumo e interpretación de datos devuelven la información a los usuarios, por ejemplo en forma de publicidad o propaganda. La memoria ROM, de este modo, aún existe, pero alejada del usuario para luego retornar reconfigurada por diversos mecanismos en la experiencia de lo digital. La mayoría de quienes construyen una cultura digital se asocian a las lógicas descritas por Ernst; sin embargo, aquella cultura no se construye simplemente por la interacción entre usuarios, sino que también está condicionada a los intereses de corporaciones para las cuales la recopilación y acumulación de datos es de vital importancia.

El concepto de archivo como mecanismo de producción de subjetividades y hegemonías se enfrenta a las nuevas prácticas culturales de la generación constante de microarchivos. En otras palabras, la tensión se establece entre los usuarios que utilizan las redes para comunicar la experiencia de sí mismos a través de información de un presente específico, y las corporaciones o instituciones de vigilancia que transan los datos de los diferentes usuarios en el mercado, explotando y acumulando la información como la materia prima del capitalismo tardío. Cabe preguntarse si acaso es esta la nueva lógica del poder arcóntico, en cuanto condiciona las estructuras sociales y el modo de presentarse a uno mismo en internet, para que cada usuario produzca, con o sin saberlo, archivos de sí generando un tipo de vigilancia y control ligado a la exterioridad, la tecnología y la comunicación mediada por aparatos electrónicos.

Las huellas y los comandos son parte de los mecanismos de subjetivación en la red que producen al sujeto-archivo digital, el cual es la condensación de ciertos datos seleccionados por algoritmos en una experiencia específica y momentánea del usuario en internet. Cada usuario se relaciona con lo digital no por medio de una subjetividad preexistente al medio, sino a través de la producción constante de sujetos-archivo digitales que finalmente son la imagen arcóntica de un momento específico de interacción con la red, la cual es una unidad en sí misma. A su vez, el sujeto-archivo digital se produce y coproduce en el consumo y la producción de datos de los usuarios conectados entre sí. Es una nueva forma de comunicación bajo las lógicas del presente del acontecimiento de la interacción lo que permite también una repetición y/o actualización de uno mismo en relación a la conectividad en entornos digitales. Es el propio sujeto-archivo digital el que encierra en sí mismo el contenido comunicacional de un momento específico. 


\section{Sujeto-archivo digital y producciones culturales digitales}

Retomando aAnn Stoler, la concepción del archivo se ha transformado desde «archive-as-source to archive-as-subject» (2010: 44); es decir, se debe comprender el archivo como un sujeto, puesto que es producido en mecanismos de subjetivación. De este modo, el concepto de subjetividad relacionado con procesos archivísticos se actualiza en lo digital para dar cabida a nuevas formas de aproximarse a estos conceptos. El sujeto, entonces, ya no es sólo un producto de fuerzas de poder que tensionan un cuerpo humano, sino que también es la configuración de un cuerpo, literalmente un corpus, por medio de la acumulación y selección de información según uno u otro interés. Así, nacen nuevas prácticas de producción literaria que precisamente problematizan la subjetividad a través de mecanismos que lo digital posibilita. Un ejemplo es la instalación desarrollada por Demian Schopf, titulada Máquina Cóndor, la cual consiste en un modelo relacional compuesto de tres operaciones consecutivas, las cuales son: a) búsqueda de 333 palabras referentes a la guerra y la economía en 12 periódicos; b) consulta y análisis en línea de los valores bursátiles de diferentes tipos de divisas y de recursos naturales importantes para la economía chilena; y c) selección de 9 palabras provenientes del léxico de la cirugía, la anatomía y la medicina forense, en la primera estrofa de un «memento mori» de Góngora. Finalmente, el ordenamiento de las 9 palabras depende de los resultados de a) y b), por lo que cada resultado es variable y situacional (demianschopf.cl/maquina-condor).

En palabras más simples: Máquina Cóndor genera automáticamente poemas según los criterios establecidos por el mismo autor. De este modo cabe preguntarse quién es el verdadero autor del resultado textual final del proceso, o si acaso puede hablarse de una subjetividad maquínica construida entre el autor, el algoritmo, el hardware y el lector/usuario. Schopf agrega al final de la descripción en su página web que el verdadero poema es «la estructura relacional dispuesta por mí y a través de la cual el mundo se expresa en tiempo real [...] el poema no es sólo una estrofa sino todos los datos sincronizados al unísono, que, por lo demás, son lo único que lo hace posible». Así, la base de datos es el componente crucial para dotar de sentido la producción generativa que sucede a través de las tres operaciones descritas. A través de la conexión a internet se configura un archivo que busca datos específicos de temas, en primera instancia, no relacionados. Sin embargo, al finalizar las operaciones, se entrega un resultado textual que posee un sentido delimitado por el archivo de su base de datos, como también por el lector activo que interactúa con la máquina. De cierto modo, la relación entre la Máquina Condor y el lector que la activa es una metonimia de la relación que cada usuario establece con las TIC. La relación es retroactiva en cuanto el resultado final es interpretado como un poema en su totalidad por el mismo lector. Los resultados imprevisibles son microarchivos 
extraídos de operaciones algorítmicas cuyo origen es la base de datos elaborada por el artista.

El lugar del usuario y su relación con las nuevas producciones y experimentaciones literarias en la red remite siempre al uso de tecnologías de comunicación y conectividad en la vida diaria. Puede incluso afirmarse que instalaciones $u$ obras como la ya mencionada configuran una estética relacional. Rocío Rueda, en su artículo «Formas de presentación de sí en Facebook: esbozos de una poética y estética dialógica», indica que

nos encontramos [...] frente a nuevas formas de comprensión de la subjetividad y sus formas de (re)presentación, (o de un representar en el sentido derridiano), de un sujeto por-venir, volcado hacia delante, de una experiencia vital en estructuras sensibles, a la espera del siguiente acontecimiento o novedad (Rueda, 2012: 213).

La (re)presentación del sujeto, o su visibilidad a través de los mecanismos de subjetivación, se relaciona con la experiencia personal del usuario en el momento propio en que sus huellas son leídas por otros o devueltas a sí mismo. Para justificar el uso de la palabra acontecimiento, hay que recurrir a lo dicho anteriormente respecto a que las lógicas de los flujos de datos y su consumo en internet suceden en un tiempo que se interpreta como el presente, el llamado «tiempo real». Es decir, la comunicación con otro es percibida como un acontecimiento en el sentido de que se trata de un evento esporádico en un presente específico donde el otro que está ausente temporal y/o espacialmente, por lo que es representado por sus huellas mediante uno $u$ otro mecanismo de las TIC. Al ser un presente en constante actualización, el evento toma mayor relevancia ya que no se contrasta con eventos pasados, sino que cada uno en sí mismo es una novedad respecto a la actualización de los sujetosarchivo digitales que se presentan para establecer el acontecimiento comunicativo. Es por ello que es necesario analizar cómo los algoritmos influyen en la construcción del sujeto-archivo digital, produciéndolo, presentándolo y actualizándolo constantemente; como también revisar el componente comunicacional y colectivo en el que se efectúa su aparición. Esto permite reflexionar sobre las nuevas relaciones entre lectores, artistas, escritores y obras digitales en una cultura digital que se caracteriza por la coproducción de subjetividades en procesos donde las tecnologías son una parte activa. En este entorno los límites de lo literario se vuelven difusos. La obra tensiona las nociones del autor y su producción poética, además de las relaciones que se establecen entre el artista, la base de datos, la máquina y el lector/usuario.

En Twitter es común encontrar operaciones similares que buscan problematizar la figura del autor en el contexto digital de los colectivos, las comunidades lectoras y la necesidad de mantener una presencia 
digital a través de la producción constante de información. Existen muchas inteligencias artificiales programadas para generar poemas y luego publicarlos en dicha plataforma de la red. Por ejemplo: en Chile, la Fábrica de bots Cyberpunga creó a Cumatron.win, el cual es un algoritmo informático programado para generar poemas de cuatro versos según una base de datos. Luego de esto, el bot los publica en Twitter. Además, en la página web https://cumatron. win/book, se genera un poemario en formato PDF que consta de cincuenta cuartillas seleccionadas aleatoriamente. De este modo cada poemario es único e irrepetible. Por su parte, en México, el colectivo poético digital brokenenglish.lol desarrolla experimentos y talleres de poesía generativa. Asimismo, cabe mencionar que este colectivo posee una estética derivada de las redes sociales y la cultura pop digital, entrecruzando la poesía con los memes, los emoticones, videos, música y lo hacker. Estos ejemplos suscitan la utilización de los conceptos relacionados con el archivo y la subjetividad para su análisis. Estas producciones utilizan mecanismos similares de recopilación de información a través de la configuración de bases de datos, además de las operaciones y pasos a seguir para generar un resultado literario. En la literatura digital las bases de la producción y el consumo literario se reconfiguran y se problematizan. ¿Quién es el verdadero autor de un poema autogenerado? ¿El resultado final es una mezcla sinsentido de palabras? ¿El lector efectivamente puede otorgarle sentido literario a aquel resultado? Reflexionar sobre el sujeto y el archivo permite una aproximación hacia las posibles respuestas de estas preguntas.

\section{El archivo, el control y el acceso}

Diversos organismos alrededor del mundo se encargan de vigilar a los usuarios de internet con mecanismos de reconocimiento de patrones y ciberespionaje. Por su parte, corporaciones ligadas a plataformas digitales venden los datos de sus usuarios, como en las redes sociales, para que sean interpretados en pos de, por ejemplo, una producción del marketing ad hoc a cada usuario particular. Los datos masivos son la materia prima de estos últimos años. Esto es un problema no sólo porque afecta la vida personal de cada ser humano conectado a internet, sino también porque se trata de procesos invisibilizados, que funcionan sin hacerse presentes directamente a la mirada del usuario. La lógica de las redes hegemónicas de comunicación en la red es la de crear la necesidad de la presencia digital, por lo que cada una es diferente y no compiten directamente entre ellas. En general no se sabe cómo funciona un aparato electrónico, simplemente se sabe cómo utilizarlo. La máquina, actualmente, no sabe que funciona, no sabe que sabe recopilar datos. Solo efectúa una acción para la cual fue programada. La preocupación 
e incluso el peligro radican en qué manera estas máquinas son programadas, bajo qué contextos políticos y económicos, y con qué fines particulares son implementadas; además de que existe un problema cuando una decisión es tomada: hasta qué punto los datos recopilados son interpretados como una verdad empírica y no como una probabilidad que debe ser estudiada. De este modo, la relación entre el ser humano y las nuevas TIC es numérica, cuantitativa, de patrones y registro de huellas. Así, los procesos de subjetivación están mediados por dichos algoritmos, los cuales son programados para construir una imagen específica y momentánea del sujeto según los datos recopilados.

Estos procesos están insertos en la utilización de ciertas páginas web, algunas más concurridas que otras, las cuales son configuradas bajo un campo de poder discursivo. El contexto de producción de las TIC y de las páginas web influye en sus modos de interacción con los usuarios. Un ejemplo es la publicidad generada «a la medida del usuario» en redes hegemónicas como Facebook o Google. Cada una de estas redes muestra en el inicio, o en la primera página de resultados, opciones que son escogidas mediante lo que se ha interpretado estadísticamente sobre los intereses de los usuarios. Puede tratarse de publicidad de agencias de viajes debido a que hace un día se hizo una búsqueda con algún elemento más o menos relacionado, como también recomendaciones según lo que ya se ha visto a lo largo de los años de utilización de una red específica. Incluso no se trata sólo de lo que uno hace activamente en las redes, sino también las conversaciones privadas, las aplicaciones que se descargan o el uso de un aparato electrónico engendran datos que se acumulan generando patrones de conducta, consumo y producción personal. Un último ejemplo es una especie de polémica sobre el gusto ligado al consumo de arte, como sería la música a través de Spotify o YouTube. Los algoritmos están programados para recomendar canciones según diversos parámetros, generalmente entre lo popular y lo anteriormente escuchado por un usuario. Así el gusto se va moldeando según las recomendaciones del algoritmo, por lo que «descubrir» nueva música es delegado a procesos informáticos ${ }^{4}$.

El sujeto-archivo digital existe en el momento de su producción, puesto que su condición de visibilidad está mediada por la manipulación y selección de datos para configurar la presencia de los usuarios, los medios y los signos que conforman la experiencia en la red. De este modo, es devuelto mediante la construcción de una unidad coherente que se ha producido con el sujeto-archivo digital como materia prima y que, a través de él, contiene un discurso. De este modo el sujeto-archivo digital no posee un tiempo más que el del presente de su producción. Su actualización es la elaboración de un sujeto-archivo diferente a cada momento, en cada plataforma web

\section{NOTAS}

4 | Como referencia incluyo tres artículos periodísticos que hablan de esta problemática:

<https://thespinoff.c-o.nz/ partner/microsoft/04-12-2019/ why-bossy-algorithms-makethe-best-dj/>,

<https://www.newscientist. com/article/mg20327156000-your-taste-in-moviessummed-up-in-an-algorithm/>, <https://observer.com/2018/09/ algorithms-take-personaltastes-hostage/>. 
y para cada uso en particular. El usuario, como sujeto inserto en un contexto sociopolítico particular, se relaciona en la red mediante la interacción con el resultado del registro de sus propias huellas. La constante producción de la experiencia digital «a la medida» de cada usuario se establece en el momento de interactuar con partes de uno mismo transformadas en diversos mecanismos, medios y discursos que son (re)presentados en la pantalla. El sujeto-archivo digital es la programación a posteriori del sujeto-usuario, como describe Mercedes Bunz, citada al comienzo de este artículo; preexiste a la interacción en la medida que la selección y manipulación es invisibilizada, registrada y reconstruida, para retornar según la necesidad y el uso que se le otorgue. El sujeto-archivo digital es consignado y reprogramado en el código numérico, en los comandos y algoritmos dentro internet.

Si bien a comienzos del siglo XXI existía un optimismo por las posibilidades que supuestamente habría de ofrecer internet a sus usuarios, hoy en día el panorama descrito se relaciona más bien a la producción de comunidades cerradas, donde el acceso a diferentes expresiones y producciones culturales se ve mermado en pos de una experiencia de lo digital relacionada al consumo y al mercado. De este modo, a pesar de ser un espacio de experimentación creativa, lo digital posee en sí mismo criterios de relevancia para diferentes plataformas. Así, las producciones artísticas y literarias compiten con producciones de masas cuya calidad se mide en categorías cuantitativas (como, por ejemplo: la cantidad de usuarios que las visitan). En consecuencia, muchas obras corren el riesgo de perderse por la problemática que suscita la necesidad de presencia digital e impacto inmediato en las redes. Para mencionar un ejemplo concreto, en Chile existe el Laboratorio de Cultura Digital, en donde uno de sus proyectos busca cartografiar obras digitales latinoamericanas. Esto debido principalmente a que muchas de aquellas obras fueron programadas en softwares que hoy en día están obsoletos.

Existen prácticas de investigación en humanidades que buscan utilizar las mismas tecnologías de archivo para desarrollar verdaderos aportes a las discusiones de hoy en día. Lev Manovich define la utilización de bases de datos en humanidades como Cultural Analytics:

we can define Cultural Analytics as the quantitative study of cultural patterns on different scales. But then we need to immediately qualify this statement. While we want to discover repeating patterns in cultural data, we should always remember that they only account for some aspects of the artifacts and their reception (manovich.net).

Es decir, se trata de la utilización de herramientas cuantitativas para definir algunos aspectos de las investigaciones, los cuales 
necesariamente deben ser analizados a su vez por procesos de reflexión y estudio. De esta manera, se puede aportar a investigaciones sobre literatura, por ejemplo, a través de la apertura de bases de datos generales, para obtener información que necesita del uso de computadoras para obtenerse. La autora anteriormente citada, Rocío Rueda, hizo en primera instancia un trabajo recopilatorio de fotografías de perfil de Facebook a través de herramientas que le permitían construir bases de datos de dicho elemento ${ }^{5}$. Así analizó las formas de presentación de los usuarios en la red social, mediante lo cual realizó sus reflexiones en torno a las estéticas dialógicas y las subjetividades «volcadas hacia delante». Este tipo de investigaciones pueden efectuarse en diversas instancias de análisis de textos literarios, no necesariamente obras digitales. Para estas últimas resulta útil en cuanto las lógicas de producción de contenido en la red tienen como base la construcción de archivos digitales.

\section{Conclusiones: sujeto-archivo digital}

El proceso al que se ha aproximado este artículo puede denominarse como subjetivación algorítmica, o la producción del sujeto por medio de algoritmos. Este proceso tiene como resultado la generación de un sujeto-archivo digital específico, el cual debe ser entendido como el conjunto de datos seleccionados por algoritmos en un contexto digital de comunicación en conjunto con el consumo y la producción de información. La problemática apunta a los nuevos modos de comunicación y consumo de datos procesados por algoritmos que recopilan datos para el funcionamiento de la red, y cómo éstos son programados bajo intereses ligados a un modelo económico que condiciona la experiencia de la red de los diferentes usuarios.

El sujeto-archivo digital descrito hasta ahora se asocia al usuario singular, pero las lógicas sociales de la cultura digital se desarrollan bajo prácticas comunicativas y de conectividad. Es necesario preguntarse cómo los algoritmos y sus procedimientos de selección y manipulación de los datos afectan y dialogan con las comunidades digitales que se conforman en internet. Además, se debe reflexionar sobre los modos de comunicación, producción y consumo que tienen como base la subjetivación algorítmica, la cual condiciona la presencia de uno mismo en la red. El sujeto-archivo digital es el producto de una relación simbiótica entre un usuario y una TIC en un contexto y tiempo específico; su configuración es lo que luego da la posibilidad de la comunicación a través de la presencia digital de los usuarios. Una de las implicancias culturales de la red es precisamente la conexión entre millones de usuarios, los que se configuran como nodos productores y receptores de datos en lógicas comunicativas

\section{NOTAS}

5 | Los resultados y reflexiones de la autora pueden observarse en su publicación «La imagen de perfil en Facebook: identidad y representación en esta red social», accesible en <https:// www.researchgate.net/ 
ya no ligadas al diálogo directo, sino basadas en la presencia digital de cada usuario. El sujeto-archivo digital produce comunidad cuando es visibilizado en una colectividad hiperconectada.

Finalmente, el archivo es hoy un concepto que remite a la información y la clasificación de documentos. Hay que analizarlo desde el contexto digital y sus mecanismos de codificación y programación. Así, lo digital como objeto de estudio no pretende responder necesariamente a viejas interrogantes, sino adecuarlas a un nuevo contexto cultural/global, donde lo expuesto en este artículo es un método de acercamiento a cuestiones tan complejas como la construcción de la subjetividad en la red y las implicancias de esto sobre la producción y el consumo de arte y literatura. Estudiar lo digital implica estudiar las sociedades y las culturas digitales, y para hacerlo es menester preguntarse por el lugar de los diferentes componentes, puesto que la tecnología como se le conoce hoy en día disloca, remece y reconfigura lo que se ha podido entender como humano, sujeto, comunidad, géneros literarios, lector, autor, entre tantos otros. 


\section{Bibliografía citada}

BUNZ, M. (2007): La utopía de la copia: el pop como irritación, Buenos Aires: Interzona Editora. CARROL, E. y ROMANO, J. (2011): Your Digital Afterlife: When Facebook, Flickr and Twitter Are Your Estate, What's Your Legacy?, Berkeley: New Riders.

DE LANDA, M. (2003): «The Archive Before and After Foucault» en Brouwer, J. y Mulder, A. (eds.), Information is alive, Rotterdam: V2 Publishing.

DERRIDA, J. (1997): Mal de archivo, Madrid: Trotta.

DELEUZE, G. y GUATTARI, F. (2002): Mil Mesetas. Capitalismo y esquizofrenia, Valencia: PreTextos.

ERNST, W. (2013): Digital Memory and the Archive, Minneapolis: University of Minnesota Press. FOUCAULT, M. (2002): Vigilar y castigar: nacimiento de la prisión, Buenos Aires: Siglo XXI Editores.

GALLAGHER, R. (2018): «Digital Subjects» en Videogames, Identity and Digital Subjectivity, Nueva York: Routledge, 1-17.

LAFUENTE, A.: «Laboratorios ciudadanos y el anarchivo de los comunes», Academia.edu, <https://www.academia.edu/14834106/Los_laboratorios_ciudadanos_y_el_anarchivo_de_los_ comunes>, [18/05/2020].

MANOVICH, L. (2017): «Automating Aesthetics», Flash Art International, 316, <https://flash--art.com/article/automating-aesthetics-lev-manovich/> [23/05/2020].

ORTEGA, É. (2019): «Zonas de Contacto: A Digital Humanities Ecology of Knowledges» en Gold, M. K. y Klein, L. F. (eds.), Debates in Digital Humanities 2019, Minneapolis: University of Minnesota Press, <https://dhdebates.gc.cuny.edu/read/4805e692-0823-4073-b4315a684250a82d/section/aeee46e3-dddc-4668-a1b3-c8983ba4d70a\#en139r>, [08/06/2020].

REY, P. y BOESEL, E. (2014): "The Web, Digital Prostheses, and Augmented Subjectivity» en Kleinman, D. y Moore, K. (eds.), Routledge Handbook of Science, Technology and Society, Nueva York: Routledge, 173-188.

RUEDA, R. (2012): «Formas de presentación de sí en Facebook: esbozos de una poética y estética dialógica», Nexus, 12, 202-215.

SCHOPF, D. (2006): Máquina Cóndor, <https://demianschopf.cl/maquina-condor>, [21/05/2020]. TELLO, A. (2018): Anarchivismo. Tecnologías políticas del archivo, Adrogué: La Cebra.

WARD, M y WISNICKI, A. (2019): "The Archive after Theory» en Gold, M. y Klein, L. (eds.), Debates in Digital Humanities 2019. Minneapolis: University of Minnesota Press, <https:// dhdebates.gc.cuny.edu/read/4805e692-0823-4073-b431-5a684250a82d/section/a8eccb81e950-4760-ba93-38e0b1f2b9d0\#ch18>, [21/05/2020]. 